\title{
Uréter ectópico como causa de pionefrosis e incontinencia urinaria
}

\author{
Martín Martín S, Torrecilla García-Ripoll JR, Sanz Ruíz A, Gonzalo Rodríguez V, Rivera Ferro J, \\ Fernández del Busto E.
}

Servicio de Urología. Hospital Clinico Universitario Valladolid.

Actas Urol Esp. 2008;32(2):256-260

\begin{abstract}
RESUMEN
URÉTER ECTÓPICO COMO CAUSA DE PIONEFROSIS E INCONTINENCIA URINARIA

El ureter ectópico se presenta aproximadamente en 1 de cada 2000 recién nacidos. En un $85 \%$ de los casos va asociado a una duplicidad pieloureteral. Las manifestaciones clínicas de esta entidad incluyen la incontinencia y las infecciones del tracto urinario. Frecuentemente el uréter ectópico se asocia a un polo superior displásico y poco funcionante. En estos casos, el tratamiento de elección es la nefrectomía polar superior. Se presenta un caso de una mujer de 23 años con dolor tipo cólico en fosa renal izquierda, fiebre y escapes urinarios. La ecografia, la urografia y la pielografia anterograda mostraron bifidez ureteral derecha, duplicidad ureteral izquierda e hidronefrosis del pielón superior izquierdo con desembocadura ectópica del mismo en la uretra. Una vez hecho el diagnóstico, se realizó una nefrectomía polar superior izquierda y ureterectomía parcial.

Palabras clave: Uréter ectópico. Duplicación ureteral. Infecciones del tracto urinario. Incontinencia urinaria. Heminefrectomía.
\end{abstract}

\section{ABSTRACT}

ECTOPIC URETER AS CAUSE OF PYONEPHROSIS AND INCONTINENCE URINARY

Ectopic ureter accounts with an incidence of 1 in 2000 newborns. When present, ectopic ureter can be associated with duplex kidneys in an $85 \%$ of the cases. Clinical manifestations of this malformation include incontinence and urinary tract infections. Ectopic ureter frequently occurs in association with a dysplastic upper pole renal moiety. When a poorly functioning upper pole segment is present, a standard surgical treatment is upper pole heminephrectomy. A 23-years old woman presented with left renal colic pain, fever and urinary leak. Ultrasound, intravenous pyelogram and antegrade pyelogram revealed a partial duplex right kidney and a complete duplex left kidney with hydronephrosis and ectopic insertion into the urethra of the left upper pole moiety. Following diagnosis upper pole heminephrectomy and partial ureterectomy was performed.

Keywords: Ectopic ureter. Ureteric duplication. Urinary tract infections. Urinary incontinence. Heminephrectomy.

$\mathrm{E}^{\mathrm{s}}$ ureter ectópico es una malformación genitourinaria, que se debe a una alteración en el origen del brote ureteral en el conducto mesonéfrico. Ocurre con mayor frecuencia en mujeres. La localización del meato ureteral y la clinica pueden ser diferentes en varones que en mujeres. La ecografia suele ser la técnica inicial en el diagnóstico, aunque la urografia ha demostrado tener mayor sensibilidad. El tratamiento del uréter ectópico asociado a una anulación del polo superior de una duplicidad ureteral es la nefrectomía polar superior con ureterectomía parcial o la nefrectomía en un sistema único. Cuando el diagnóstico es temprano y la función del polo superior está conservada pueden llevarse a cabo técnicas quirúrgicas más conservadoras ${ }^{1,3,4}$.

\section{CASO CLÍNICO}

Se trata de una mujer de 23 años de edad, sin alergias medicamentosas conocidas, que acude al Servicio de Urgencias por presentar dolor tipo cólico en fosa renal izquierda, irradiado a genitales 
y acompañado de nauseas y fiebre. La paciente refiere incontinencia urinaria con goteo discontinuo, acompañado de micciones normales desde la infancia y cólicos nefríticos izquierdos de repetición a lo largo del último año.

A la exploración física presenta una temperatura axilar de $38,8^{\circ} \mathrm{C}$, una tensión arterial de 125/70 mmHg. El abdomen es blando, depresible, con ligeras molestias a la palpación en el trayecto ureteral izquierdo. No se aprecian masas ni megalias. La puñopercusión renal izquierda es positiva y los genitales externos normales.

Exploraciones complementarias: Hemograma: hemoglobina $13,8 \mathrm{~g} / \mathrm{dl}$, leucocitos $17250 / \mathrm{mm}^{3}$ (83\% de neutrófilos). Bioquímica: creatinina 0,7 $\mathrm{mg} / \mathrm{dl}$. Sistemático de orina: hematuria, piuria. En la radiografia simple de Abdomen no se distinguen imágenes compatibles con litiasis. La ecografia muestra la sospecha de duplicidad pielocalicial bilateral, con dilatación de pelvis y ureter correspondiente a pielón superior izquierdo. Con la pielografia intravenosa comprobamos la presencia de bifidez ureteral derecha y un pielón inferior izquierdo desplazado hacia abajo y hacia fuera por efecto masa, con la pelvis y porción superior de uréter más alejados de la columna vertebral que en el lado contrario (Fig. 1).

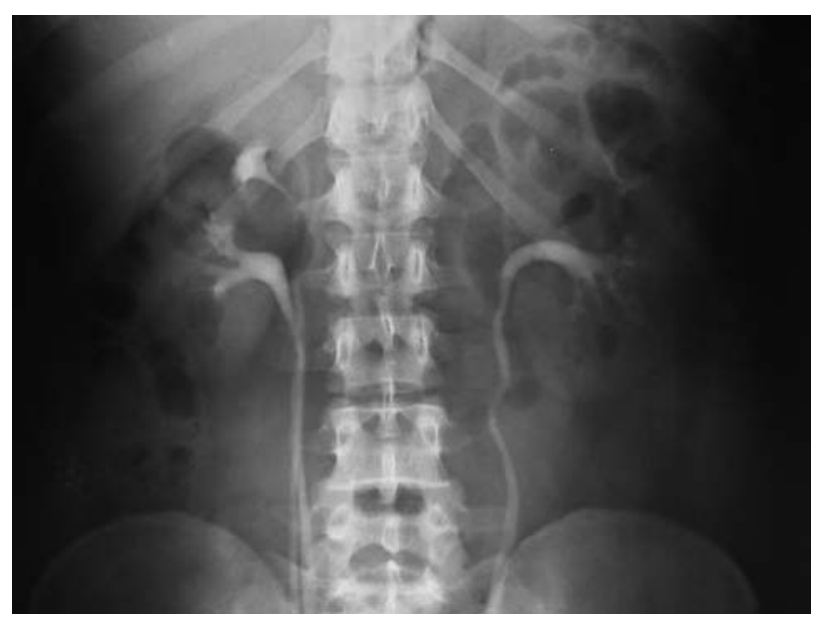

FIGURA 1. La urografía nos muestra una bifidez ureteral derecha y un pielón inferior izquierdo desplazado hacia abajo y hacia fuera por efecto masa.

Se decide el ingreso practicándose punción percutánea del pielón superior izquierdo, observando gran dilatación del mismo (Fig. 2) y extrayendo orina purulenta (E. coli en urocultivo), por

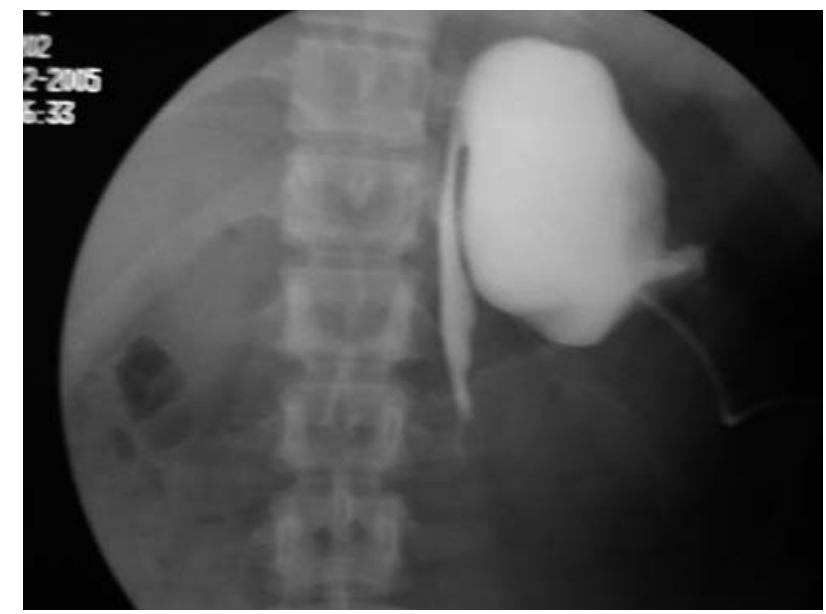

FIGURA 2. Punción percutánea del pielón superior izquierdo.

lo que se deja colocada una sonda de nefrostomía y se instaura tratamiento antibiótico. Tras la descarga del pielón y comprobada la ausencia de orina purulenta en su interior se introduce contraste observando un megauréter dependiente de dicho pielón superior dilatado, que desemboca de manera ectópica en uretra.

Una vez que el cuadro agudo ha cedido y con estudios preoperatorios dentro de la normalidad, la paciente es intervenida quirúrgicamente realizándose nefrectomía polar superior izquierda y ureterectomía parcial hasta cercanías de la vejiga (Fig. 3). La paciente tras la cirugía evoluciona favorablemente, con desaparición de su incontinencia urinaria. En urografia intravenosa de control (Fig. 4), practicada al mes de la intervención, se observa buen funcionamiento del hemirriñón izquierdo, persistiendo la bifidez en lado derecho.

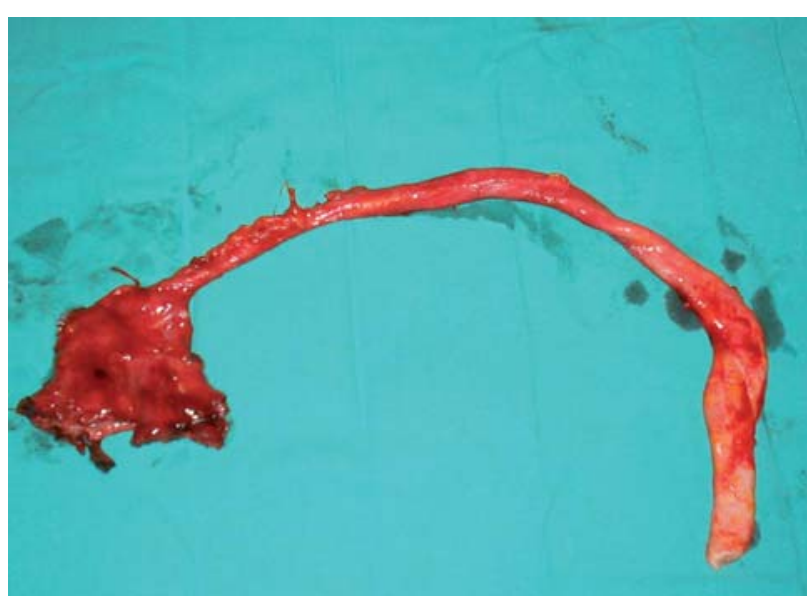

FIGURA 3. Pieza correspondiente a la extracción quirúrgica del tejido disgenésico renal y parte del uréter. 


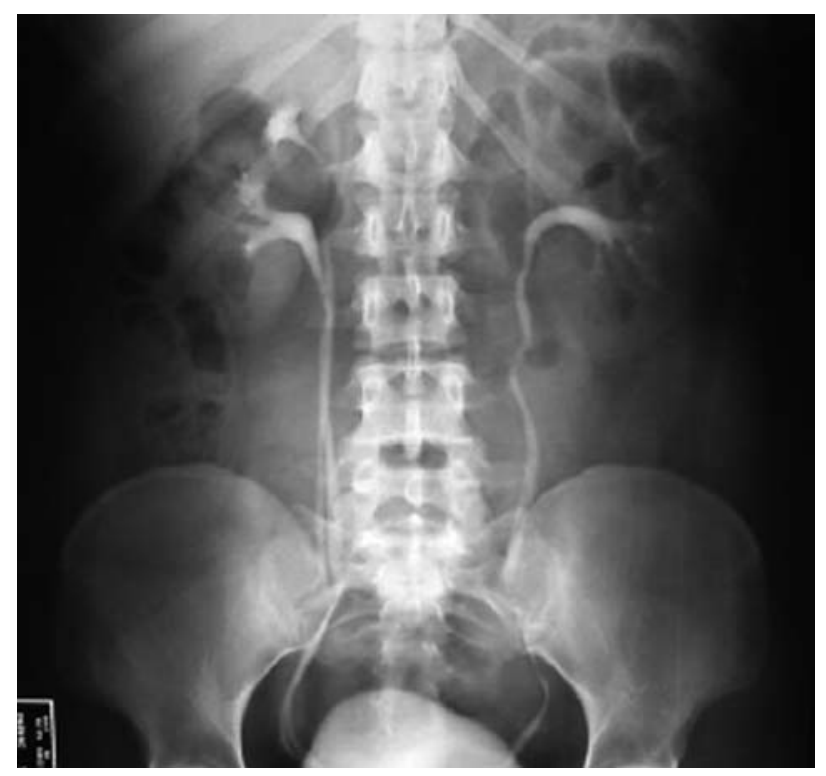

FIGURA 4. En urografía de control, pasado un mes de la intervención, se observa el buen funcionamiento del hemirriñon izquierdo y la bifidez ureteral en el lado derecho.

\section{DISCUSIÓN}

A la cuarta semana semana de gestación surge una invaginación de la porción distal del conducto mesonéfrico. Esta invaginación es el brote ureteral, que interactúa con una masa de mesénquima que constituye el blastema metanéfrico. El segmento de conducto mesonéfrico distal al brote ureteral es el conducto excretor común. Este conducto finalmente se incorpora a la vejiga en desarrollo y se convierte en parte del trígono. Una alteración en el origen del brote ureteral da lugar a una localización anómala del orificio ureteral. Por lo tanto, el meato ureteral se considera ectópico, cuando desemboca en un lugar distinto del correspondiente a su posición normal en el trígono.

El brote ureteral se alarga progresivamente y después se bifurca a alguna distancia de su punto de origen, al contacto con el metanefros da lugar a la pelvis y a los cálices. Cuando la división es precoz, a poca distancia del conducto mesonéfrico, su aspecto es el de una bifidez. Por el contrario, si a partir del conducto mesonéfrico se originan dos brotes ureterales, se producen dos interacciones separadas y completas entre el uréter y el blastema metenéfrico. Como resultado se forman dos unidades renales con sistemas colectores, uréteres y orificios ureterales separados. E1 orificio del polo inferior es más cefálico y externo que el orificio del polo superior, más caudal e interno. Para alcanzar estas localizaciones los dos uréteres y los dos orificios rotan 180 grados en sentido horario sobre sus ejes longitudinales. Esta disposición, casi constante, fue descrita por Weigert (1877) y Meyer (1946) por lo que lleva la forma de ley de estos autores ${ }^{1,2}$.

En la mayoría de los casos, la duplicidad ureteral es un hallazgo incidental en sujetos asintomáticos. Sin embargo, es importante recordar que hay tres complicaciones asociadas que en orden de frecuencia son: el reflujo vesicoureteral, el ureterocele y el uréter ectópico. Según el desarrollo del presente caso, nos centraremos en este último ${ }^{3}$.

La ectopia ureteral se presenta aproximadamente en uno de cada dos mil recién nacidos. Por sexos es más frecuente en el sexo femenino (85\%), presentándose en el $10 \%$ de los casos de forma bilateral. El 80\% de los meatos ectópicos se asocia con un sistema colector duplicado, y frecuentemente el polo superior es displásico y poco funcionante 4

El uréter ectópico en una duplicación ureteral completa puede insertarse en cualquier parte a lo largo del "ectopic pathway" o "sendero ectópico", el cual es diferente dependiendo del sexo ${ }^{3}$. En las mujeres los sitios más frecuentes son la uretra (35\%), el vestíbulo vaginal (34\%) y la vagina (25\%) mientras que en los varones predomina la desembocadura en uretra posterior (58\%), la vesícula seminal (26\%) y el conducto eyaculador (15\%). Cuanto más lejano está el orificio ureteral de su posición habitual, mayor es el grado de anormalidad del desarrollo renal ${ }^{4,5}$.

Las presentaciones clásicas de esta patología son la infección urinaria, como principal síntoma, la hidronefrosis, la pionefrosis (consecuencia final de la retención purulenta pielocalicial por obstrucción del meato ectópico, que conlleva la destrucción parenquimatosa renal) y la incontinencia urinaria. Al igual que la localización, la presentación clínica también puede ser diferente en varones que en mujeres. Las niñas suelen presentar infecciones urinarias agudas o de repetición, incontinencia urinaria que suele ser congénita, permanente e incompleta, secreción vaginal o dolor cólico ${ }^{1}$. 
En los niños la inserción ureteral ectópica suele estar por encima del esfinter externo de la uretra, por lo que no suelen presentar incontinencia, aunque hay estudios que revelan varones con dicha sintomatología ${ }^{6}$. Cuando el uréter drena en una vesícula seminal puede provocar epididimitis de repetición ${ }^{7}$.

El diagnostico ecográfico prenatal se produce en el $58 \%$ de los $\operatorname{casos}^{8}$. La detección en otras edades de la vida se lleva a cabo mediante la clínica del paciente y las técnicas de imagen.

Aunque la ecografia suele ser la técnica inicial en el diagnóstico, hay estudios que demuestran que tiene menos sensibilidad que la urografia intravenosa para detectar tanto la duplicidad pieloureteral, como el ureter ectópico. ${ }^{4}$

La urografia intravenosa nos confirma el diagnóstico en la mayoría de los casos, aunque nos presenta signos indirectos, puesto que el polo superior es poco funcionante. Innes Williams propuso 5 criterios para poner en evidencia "un pielón superior mudo ${ }^{1,9 ":}$

- El número de cálices es inferior al normal y el cáliz superior visible está separado de la sombra superior del riñón más de de $40 \mathrm{mms}$.

- El polo superior del pielón visible está desplazado hacia abajo y afuera, dando el aspecto típico de "flor marchita".

- El cáliz superior es corto y sus ramas evocan el diagnóstico de malrotación.

- La pelvis está desplazada hacia fuera a distancia de la columna vertebral, y sólo se aproxima a ésta a la altura de la quinta vértebra lumbar.

- El uréter visible retiene mucho más tiempo el contraste.

La pielografia anterógrada, previa punción percutánea translumbar nos ayudará a estudiar el pielón no funcionante, ya sospechado por urografia y ecografia ${ }^{1}$.

En caso de duda, y ante una elevada sospecha clínica la resonancia magnética puede ser útil a la hora de aclarar el diagnóstico ${ }^{10}$.

El tratamiento del uréter ectópico asociado a una anulación del polo superior de una duplicidad renal es la nefrectomía polar superior con ureterectomía parcial o la nefrectomía en un sistema único ${ }^{1,3,11-15}$. La tasa de reoperaciones por patología asociada al muñón ureteral redundante (infecciones urinarias, ureterocele), es del $8 \%$, por lo que el riesgo importante de lesionar el uréter sano puede sobrepasar los beneficios de completar la ureterectomía ${ }^{8}$. El descenso medio de la función renal, utilizando DMSA-Tc99, después de dicha cirugía es del $6,8 \%{ }^{11}$. Este tipo de cirugia también puede llevarse a cabo por vía laparoscópica, ya sea transperitoneal (la más usada) como retroperitoneal. Esta vía de abordaje supone una menor estancia hospitalaria, una mayor magnificación del campo quirúrgico, un mejor resultado estético y un menor tiempo de recuperación, pero se aumenta claramente el tiempo quirúrgico ${ }^{12-15}$. También se han descrito técnicas quirúrgicas minimamente invasivas con buenos resultados como la embolización arterial selectiva del riñón afectado con espuma de alcohol polivinilo ${ }^{16}$. Cuando el diagnostico es temprano y el polo superior del riñón no presenta hidronefrosis marcada, con una función aceptable pueden llevarse a cabo técnicas quirúrgicas más conservadoras: la reimplantación urétero-vesical, anastomosis pielo-piélica con ligadura del ureter ectópico, la anastomosis urétero-ureteral o la dilatación endoscópica del meato ureteral obstruido ${ }^{1,3,4,17}$.

\section{REFERENCIAS}

1. Fernández del Busto E, et al. Manual de Urología general. $3^{a}$ ed. Universidad de Valladolid, Secretariado de Publicaciones e Intercambio Editorial. 2004;126-50.

2. Campbell. Tratado de Urología. $8^{\mathrm{a}}$ ed. Editorial Médica Panamericana. 2004;2192-238.

3. Ahmed S, Morris LL, Byard RW. Ectopic ureter with complete ureteric duplication in the female child. $J$ Pediatr Surg. 1992;27(11):1455-1460.

4. Grande Moreillo C, Rodó Salas J, Morales Fochs L. Uréter ectópico como causa de de incontinencia urinaria en las niñas. Actas Urol Esp. 2000;24(4):314-318.

5. Lee G, Attar K, Hudd C. Case report: Minimally invasive treatment of an unusual obstructive ectopic upper pole ureter. Int Urol Nephrol. 2004;36(1):21-22.

6. Zuniga ZV, Guzzo T, Docimo SG. Bilateral single system ectopic ureters in male infant. Urology. 2005;66(2):432.

7. Pascual Samaniego M, Egea Camacho J, Cortiñas González JR, Calleja Escudero J, Rivero Martínez MD, Fernández del Busto E. Agenesia renal derecha y abocamiento ectópico del uréter en dilatación quística de la vesícula seminal. Actas Urol Esp. 2004;28(9):688-693.

8. Ade-Ajayi N, Wilcox DT, Duffy PG, Ransley PG. Upper pole heminephrectomy: is complete ureterectomy necessary?. BJU Int. 2001;88(1):77-79.

9. Williams ID. Urology in childhood. Enciclopedia of Urology. Berlin Springer Verlag. Vol 15. 353 PP. 1958.

10. Krishnan A, Baskin LS. Identification of ectopic ureter in incontinent girl using magnetic resonance imaging. Urology. 2005;65(5):1002. 
11. Gundeti MS, Ransley PG, Duffy PG, Cuckow PM, Wilcox DT. Renal outcome following heminephrectomy for duplex kidney. J Urol. 2005;173(5):1743-1744.

12. Dubosq F, Doublet JD, Traxer O, Haab F, Amarenco G. Incontinence from ectopic ureter: retroperitoneal laparoscopic urovascular exclusion of upper pole. J Urol. 2004;171(2 Pt 1):800-801.

13. Kawauchi A, Fujito A, Naito Y, Soh J, Ukimura O, Yoneda $\mathrm{K}$, et al. Retroperitoneoscopic heminephroureterectomy for children with duplex anomaly: Initial experience. Int $\mathrm{J}$ Urol. 2004;11(1):7-10.

14. Wang DS, Bird VG, Cooper CS, Austin JC, Winfield HN Laparoscopic upper pole heminephrectomy for ectopic ureter: initial experience. Can J Urol. 2004;11(1):21412145 .

15. Jednak R, Kryger JV, Barthold JS, González R. A simplified technique of upper pole heminephrectomy for duplex kidney. J Urol. 2000;164(4):1326-1328.
16. Solinas A, De Giorgi F, Frongia M. Embolization of a hypoplastic kidney with a vaginal ectopic ureter in a case of pseudo-incontinence. Arch Ital Urol Androl. 2004;76(3): 117-118.

17. Akbal C, Lee SD, Kaefer M. Minimally invasive retrograde endourological technique for obstructed ectopic ureter. J Urol. 2004;172(4 Pt 1):1445.

Correspondencia autor: Dr. S. Martín Martín Servicio de Urología

Hospital Clínico Universitario de Valladolid

Ramón y Cajal, s/n - 45005 Valladolid

Tel.: 983420000

E-mail: risimartin7@hotmail.com

Información artículo: Nota Clínica

Trabajo recibido: julio 2006

Trabajo aceptado: septiembre 2006 